\title{
Sciendo
}

\section{RNA-SEQ STUDY OF HEPATIC RESPONSE OF YELLOW-FEATHER CHICKENS TO ACUTE HEAT STRESS}

\author{
Quan Zhang ${ }^{1,2}$, Yi Kang Luo ${ }^{1}$, Bo Hai Zhang ${ }^{1}$, Yan Zi Chan', Lin Lin Huang ${ }^{1}$, Yin Wang ${ }^{1}$, \\ Jia Ming Liang ${ }^{1}$, Xi Quan Zhang ${ }^{2}$ \\ 'Department of Animal Science, Agricultural College, Guangdong Ocean University, 524088, \\ Zhanjiang, China \\ ${ }^{2}$ Guangdong Provincial Key Laboratory of Agro-animal Genomics and Molecular Breeding, South \\ China Agricultural University, 510642, Guangzhou, China \\ •Corresponding author: zhang_quan@cau.edu.cn
}

\begin{abstract}
The yellow-feather broiler is a popular poultry breed in Asia, particularly in China. In this study, we performed RNA-seq analysis to identify differentially expressed genes (DEGs) in the liver of yellow-feather broilers that had been subjected to acute heat stress treatment $\left(38 \pm 1^{\circ} \mathrm{C}\right.$ for $4 \mathrm{~h}$, recovery $\mathbf{2} \mathrm{h}$ ) and determine the response of the liver to high temperature and its effects on yellowfeather broiler physiology. We found that the cloacal temperature and respiratory rate of yellowfeather chickens were significantly increased immediately after the initiation of acute heat stress $\left(38^{\circ} \mathrm{C}\right)$ treatment. And after recovery for $2 \mathrm{~h}$, there was no difference in the cloacal temperature and respiratory rate between the acute heat stress and control groups. A total of 834 DEGs were observed in response to heat stress by RNA-seq. Almost half of the DEGs were involved in the lipid and energy metabolism, including fatty acid metabolism (ACOX1, ACACA, ACSL1, ACSL6, $A C A A 1, A C A A 2, H A D H B$, and $F A S N$ ) and propanoate metabolism (ACSS2, ALDH2, ACACA, $D L A T, A L D H 7 A 1, M D H 1, M E 1, A B A T, S U C L G 2$, and ACSS3). Our findings provide the context for RNA-seq studies in the liver of yellow-feather chickens and suggest that the liver of yellowfeather broilers has the lipid and energy metabolism physiological mechanisms activated in response to heat stress.
\end{abstract}

Key words: yellow-feather chickens, liver, heat stress, RNA-seq

Global warming is affecting the broiler chicken industry worldwide (Windhorst, 2007). In the past few decades, broiler production performance, particularly in terms of growth rates, has been significantly improved through genetic selection (Deeb and Cahaner, 2002; Mckay et al., 2000). However, high environmental temperatures have a more detrimental effect on fast-growing broilers than on slow-growing broilers (Cahaner and Leenstra, 1992; Cheng et al., 2018; Lu et al., 2007), particularly with regards to the body weight of these chickens (Rimoldi et al., 2015; Sohail et al., 
2012). Liver, which performs important functions in maintaining the homeostasis of energy metabolism, is more sensitive than other organs to acute heat stress in broilers (Huang, 2017; Hubbard et al., 2018; Jastrebski et al., 2017; Lin et al., 2006; Tang et al., 2015; Xie et al., 2013) and is, thus, an ideal candidate tissue for studying the effects of this stress on lipid and energy metabolism.

The yellow-feather broiler is a popular poultry breed in Asia, particularly in China, that is known for its slower growth than commercial broilers and unique meat flavor. Previous studies on chickens have shown that fast-growing broilers liver transcriptome responded to treatment with cyclic high ambient temperature and observed changes in metabolic, physiologic, and cellular responses (Coble et al., 2014; Jastrebski et al., 2017; Lan et al., 2016). Study of heart RNA-seq of yellow-feather chickens subjected to heat stress showed that the heart of these chickens has specific physiological mechanisms for regulating body growth under heat stress (Zhang et al., 2019). However, to date, there has been limited research on the response of yellow-feather broilers liver to heat stress. In the present study, we investigated the expression of genes in the liver of yellow-feather chickens with regards to the response to acute heat stress and analyzed the effects of heat stress on the metabolism of these birds.

\section{Material and methods}

\section{Experimental design and management of animals}

The 36 male yellow-feather chickens (65-day-old) were raised in two environmentally controlled rooms at the Poultry Breeding Center of Guangdong Ocean University, each of which was divided into three pens. Each pen contained six cocks, which were acclimated to the environment for 5 days and had access to feed and water ad libitum. Seventy-day-old yellow-feather chickens in one of environmentally controlled rooms were subjected to heat stress for $4 \mathrm{~h}$ at $38^{\circ} \mathrm{C}$ and $55 \%$ relative humidity (the $4 \mathrm{HR} 0$ group), followed by recovery for $2 \mathrm{~h}$ at $25^{\circ} \mathrm{C}$ and $55 \%$ relative humidity (the 4HR2 group). The yellow-feather chickens in another environmentally controlled room were kept at $25^{\circ} \mathrm{C}$ and $55 \%$ relative humidity (the control group). All the birds were cared for and treated in accordance with the guidelines provided by the Guangdong Ocean University Animal Care and Use Committee (permit number: SYXK 2014-0053).

\section{Phenotypic measurements and sample collection}

The respiratory rate of each cock was determined by counting the number of thoracic breaths per minute. The cloacal temperature of each broiler was measured using a digital thermometer (accurate to $0.1^{\circ} \mathrm{C}$ ), until the reading was stable. The digital thermometer was inserted into the cloaca of each cock to a depth of approximately $2.5 \mathrm{~cm}$. The cocks were euthanized before heat treatment ( $\mathrm{n}=6$, control), after four hours of acute heat $4 \mathrm{HR} 0(\mathrm{n}=6)$ and after 2 hours of recovery in thermo-neutral conditions 4HR2 $(n=6)$, and 18 samples of liver tissue were collected and stored at 
$-80^{\circ} \mathrm{C}$ until required. Three of six liver samples of each treatment were selected at random for RNA-seq.

\section{Transcriptome profile in response to acute heat stress}

We used an Illumina HiSeq 2500 platform to identify the differentially expressed genes (DEGs) in the liver samples collected from yellow-feather chickens that were subjected to acute heat stress. For sequencing, total RNA was extracted from the liver samples using TRIzol reagent (Invitrogen, catalog no. 15596026, USA), according to the manufacturer's instructions. An NEBNext ${ }^{\mathbb{R}}$ Ultra $^{\mathrm{TM}}$ RNA Library Prep Kit for Illumina ${ }^{\circledR}$ (NEB, USA) was used to prepare the sequencing libraries, according to manufacturer's recommendations. The sequencing reads were mapped to the Gallus gallus genome assembly (ftp://ftp.ensembl.org/pub/release-92/fasta/gallus_gallus/dna/) using HISAT2 v2.0.4 (Kim et al., 2015). HTSeq v0.9.1 was used to count the reads (Anders et al., 2015). The gene expression levels were quantified as fragments per kilobase of transcript sequence per million base pairs sequenced (fragments per kilobase million; FPKM) (Trapnell et al., 2010). The DESeq R package (1.18.0) (Anders and Huber, 2010) was used to analyze the DEGs in the heat stress liver samples (P-value $<0.05$ and false discovery rate $(\mathrm{FDR})<0.05$ were used as the threshold values). In a simulation study evaluating DESeq the DEG detection was above $80 \%$ for $n=3$ and $\log 2$ foldchange $\geq 1.0$ but at a cost of higher than in other tools false discovery rate (Schurch et al., 2016). The GOseq R package (http://bioinf.wehi. edu.au/software/goseq/) (Young et al., 2010) and KOBAS software (http://kobas. cbi.pku.edu.cn) (Mao et al., 2005) were used to analyze the results of gene ontology (GO) and Kyoto Encyclopedia of Genes and Genomes (KEGG) enrichment analysis.

\section{Quantitative reverse transcription-polymerase chain reaction and statistical analysis}

We used quantitative reverse transcription-polymerase chain reaction (qRT-PCR) analysis to determine the expression levels of six genes (HSPA2, ACACA, FASN, $A C S L 1, A C O X 1$, and $A C A A 1$ ). The sequences of the primer pairs used for amplification of the selected genes are shown in Table 1, GAPDH was used as a reference housekeeping gene. The $2^{-\Delta \Delta C T}$ method (Livak and Schmittgen, 2001) was used to analyze the gene expression levels determined by qRT-PCR. The values obtained for respiratory rate, body temperature, and mRNA expression levels were analyzed using one-way analysis of variance (ANOVA) with SPSS software (v19.0, IBM, USA).

Table 1. Information on primers for qRT-PCR

\begin{tabular}{lll|lll}
\hline Gene name & \multicolumn{1}{c|}{ Gene ID } & \multicolumn{1}{c}{ Forward primer } & Reverse primer & Length \\
\hline ACOX1 & NM_001006205 & attcatcctcaacagccca & ctgagtgtagagctgagcca & $103 \mathrm{bp}$ \\
ACACA & NM_205505 & tgagtgccttcgagttgtga & acatcaccactgcaaacacc & $109 \mathrm{bp}$ \\
ACSL1 & NC_006091 & gcacccttccgacaatacc & tgcattgctaagtcacacgg & $112 \mathrm{bp}$ \\
ACAA1 & NM_001197288 & gagaatggcagcactacagc & ctccaaccacagcaaaggac & $139 \mathrm{bp}$ \\
FASN & NC_006105 & ctgatgggatttgggccac & cccctccaataagcagcaag & $100 \mathrm{bp}$ \\
HSPA2 & NM_001006685 & ttgataagggccagatccag & ttgatagggccagatccag & $105 \mathrm{bp}$ \\
GAPDH & M_11213.1 & tagtgaaggctgctgctgat & aaggtggaggaatggctgtc & $103 \mathrm{bp}$ \\
\hline
\end{tabular}




\section{Data deposition}

The RNA-seq data were submitted to the Sequence Read Archive (SRA) at the National Center for Biotechnology Information (NCBI) under the accession number PRJNA490069 (https://www.ncbi.nlm.nih.gov/sra/PRJNA490069).

\section{Results}

\section{Phenotypic measurements}

The cloacal temperature and respiratory rate of yellow-feather chickens were significantly increased immediately after the initiation of acute heat stress $\left(38^{\circ} \mathrm{C}\right)$ treatment $\left(\mathrm{P}<0.05\right.$; Figure 1). The average cloacal temperature reached $46^{\circ} \mathrm{C}$ in $4 \mathrm{~h}$ of acute heat stress treatment, whereas the respiratory rate began to decrease. After recovery for $2 \mathrm{~h}$, there was no difference in the cloacal temperature and respiratory rate between the acute heat stress and control groups.
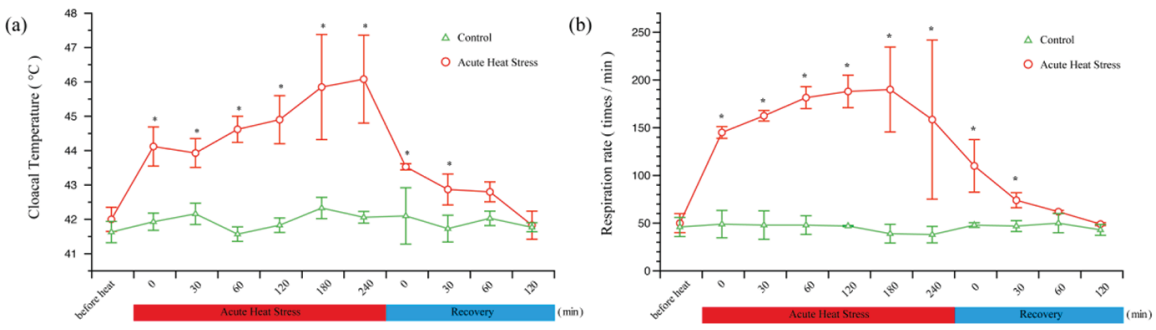

Figure 1. Cloacal temperature (a) and respiratory rate (b) of acute heat stress and control yellow-feather chickens during the $4 \mathrm{~h}$ heat stress and $2 \mathrm{~h}$ recovery periods. The asterisk indicates the significant difference of each detected time between the heat stress and control groups $(\mathrm{P}<0.05)$

\section{Analysis of differential gene expression}

The total number of clean reads varied from 46 to 73 million, and approximately $90 \%$ of these reads were mapped onto the reference genome of chicken (Table 2). Some differences in read counts were detected between biological replicates, especially between Control_1 and 4HR2_3. The quantity of total mRNA analyzed was identical in these samples, indicating that the different read counts may have arisen during processing. However, this discrepancy did not significantly affect gene expression analysis because the FPKM values were corrected for the total number of read counts for each sample. Approximately $75 \%$ of the reads were mapped to the exons (Figure 5). A total of 834 DEGs were observed in response to acute heat stress in the liver samples of the 4HR0 and 4HR2 groups. The number of DEGs in the 4HR2 group $(n=786)$ was 7 times higher than that of the 4 HR0 group $(n=103$, Figure 2). This shows that during the period of recovery from acute heat stress, liver produces a strong response, which is manifested in the different number of DEGs under acute heat stress and recovery conditions. Cluster analysis of DEGs was used to determine the relationship between the acute heat stress and control groups (Figure 6). Of these 834 genes, 481 were down-regulated and 353 were up-regulated 
(Figure 2). A total of 45 down-regulated DEGs and 10 up-regulated DEGs, respectively, were shared with the 4HR0 and 4HR2 groups. According to DEGs GO and KEGG analysis results, six DEGs (HSPA2, ACACA, FASN, ACSL1, ACOX1, and $A C A A 1$ ) associated with fatty acid metabolism and HSP were selected for validation by qRT-PCR. The analysis of the expression levels of six DEGs results were shown in Table 3. The results of qRT-PCR were highly correlated with the FPKM values estimated by RNA sequencing $(r=0.99)$.

Table 2. Number of reads before and after FastQC filtering, and number of mapped and uniquely mapped reads

\begin{tabular}{l|c|c|c|c}
\hline cDNA library & Raw reads & Clean reads & Mapped reads & Uniquely mapped \\
\hline Control_1 & $75,770,336$ & $73,606,478$ & $65,859,010$ & $62,887,732$ \\
Control_2 & $67,629,042$ & $65,974,694$ & $58,986,419$ & $56,409,777$ \\
Control_3 & $60,102,230$ & $58,707,790$ & $53,203,729$ & $51,025,529$ \\
4HR0_1 & $58,486,260$ & $56,919,196$ & $50,959,137$ & $48,798,262$ \\
4HR0_2 & $63,741,388$ & $62,248,222$ & $56,567,826$ & $54,336,101$ \\
4HR0_3 & $62,232,272$ & $60,889,198$ & $55,359,485$ & $53,077,675$ \\
4HR2_1 & $64,954,228$ & $63,327,084$ & $56,476,671$ & $54,254,204$ \\
4HR2_2 & $64,615,270$ & $63,013,114$ & $57,369,907$ & $54,949,809$ \\
4HR2_3 & $48,202,070$ & $46,718,578$ & $41,835,339$ & $40,175,531$ \\
Average & $62,859,392$ & $61,267,150$ & $55,179,724$ & $52,879,402$ \\
\hline
\end{tabular}

$4 \mathrm{HR} 0$ group was subjected to acute heat stress at $38^{\circ} \mathrm{C}$ for $4 \mathrm{~h}, 4 \mathrm{HR} 2$ group was subjected to recovery for $2 \mathrm{~h}$ at $25^{\circ} \mathrm{C}$ after acute heat stress, and control group was maintained at $25^{\circ} \mathrm{C}$ during the experiment period. There are three biological repetitions for each group.

\section{GO and KEGG pathway analysis of the DEGs}

We performed GO enrichment analysis of the DEGs using the GOseq R package to identify the biological processes related to acute heat stress. The significantly $($ FDR $<0.05)$ enriched GO terms for the DEGs in the liver samples are represented in Figure 3. These GO terms included biological process (BP), cellular component (CC), and molecular function (MF) terms.

We detected 48 significantly enriched GO terms for the 4HR0 and 4HR2 groups. Among these, 37, 4, and 7 terms were related to $\mathrm{BP}, \mathrm{CC}$, and $\mathrm{MF}$ categories, respectively. The BP categories related to metabolic, catabolic, and biosynthetic processes in the liver were enriched. The metabolic processes included metabolic progress $(n=422)$, single-organism metabolic progress $(n=225)$, small molecule metabolic progress $(n=112)$, lipid metabolic progress $(n=71)$, and fatty acid metabolic progress $(n=28)$. The catabolic processes included organic acid catabolic process $(n=25)$, carboxylic acid catabolic process $(n=25)$, and cellular amino acid catabolic process $(n=15)$. The biosynthetic processes included organic acid biosynthetic process $(n=21)$, carboxylic acid biosynthetic process $(n=21)$, and small molecule biosynthetic process $(n=28)$. We also noticed enrichment of the oxidation-reduction process $(n=86)$ term. The results of GO enrichment analysis showed that in liver, $50.6 \%$ DEGs were mainly involved in metabolism, under acute heat stress. A total of $43 \mathrm{GO}$ terms were found to be enriched in the 4HR2 group. 


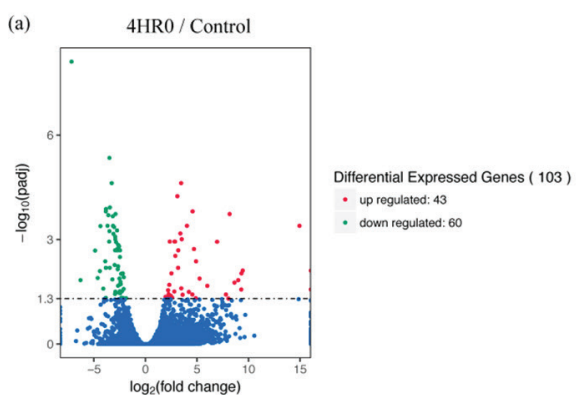

(c)

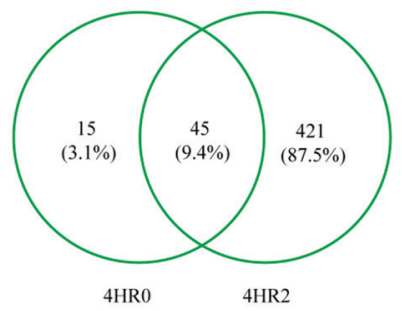

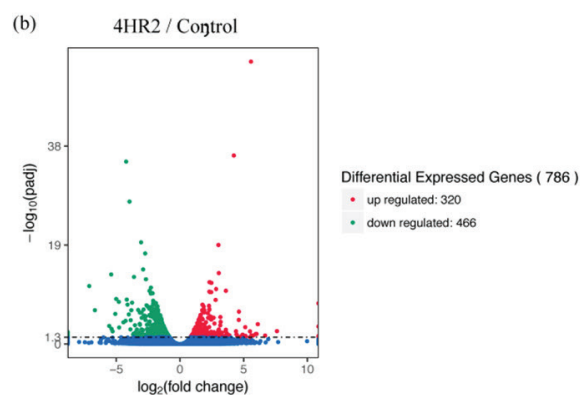

(d)

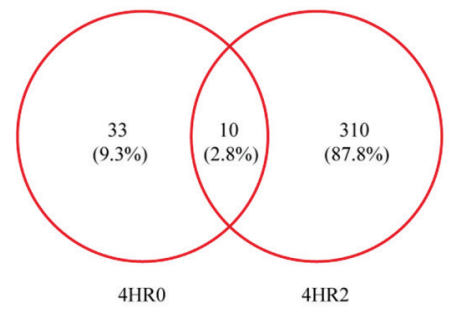

Figure 2. Volcano plot and venn diagram of differentially expressed genes (DEGs) in 4HR0 and 4HR2 groups compared with control group, respectively. Volcano plot DEGs in 4HR0 and 4HR2 group were displayed in panel a and panel b, respectively. Y-axis: the mean gene expression value of $\log _{10}(\mathrm{FDR})$. Xaxis: the $\log 2$ fold change value of gene expression. The red and green dots represent up- and down-regulated DEGs, respectively (FDR $<0.05)$. Venn diagram of up- and down-regulated DEGs were displayed in panel $\mathrm{c}$ and panel d, respectively. (c) There were 421 (87.5\%) and $15(3.1 \%)$ down-regulated DEGs in 4HR2 and 4HR0 group, respectively. And $45(9.4 \%)$ down-regulated DEGs were shared with 4HR2 and 4HR0 groups. (d) There were $310(87.8 \%)$ and 33 (9.3\%) up-regulated DEGs in 4HR2 and 4HR0 group, respectively. And ten (2.8\%) up-regulated DEGs were shared with 4HR2 and 4HR0 groups. 4HR0 group was subjected to acute heat stress at $38^{\circ} \mathrm{C}$ for $4 \mathrm{~h}, 4 \mathrm{HR} 2$ group was subjected to recovery for $2 \mathrm{~h}$ at $25^{\circ} \mathrm{C}$

after acute heat stress, and control group was maintained at $25^{\circ} \mathrm{C}$ during the experiment period

The KEGG pathway analysis (Kanehisa and Goto, 1999) of the DEGs was performed to characterize the functional consequences of changes in gene expression in the liver under acute heat stress and recovery. It was observed that the DEGs in the 4HR0 and 4HR2 groups were enriched in fatty acid metabolism $(\mathrm{n}=5$ and $\mathrm{n}=16$, respectively) and in the biosynthesis of unsaturated fatty acids ( $\mathrm{n}=4$ and $\mathrm{n}=11$, respectively) (Figure 4). The steroid biosynthesis $(\mathrm{n}=3)$ and caffeine metabolism $(n=2)$ genes were enriched in the 4HR0 group (Figure 4 A). The pathways of pyruvate metabolism $(n=11)$, glycine, serine, and threonine metabolism $(n=11)$, propanoate metabolism $(n=8)$, and metabolic pathways $(n=105)$ were enriched in the 4HR2 group. In addition, genes of glycolysis/gluconeogenesis $(n=14)$ and peroxisome $(n=14)$ were also enriched in the 4HR2 group (Figure 4 B). The results of KEGG pathway analysis also showed that DEGs were mainly involved in metabolism in liver under acute heat stress. Moreover, the biochemical pathways altered in the $4 \mathrm{HR} 2$ group were more complex than those in the 4HR0 group. 
A.

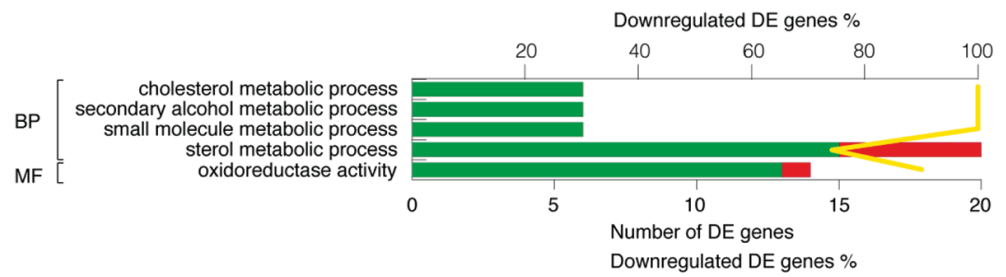

B.

BP

oxidation-reduction process carboxylic acid metabolic process small molecule metabolic process oxoacid metabolic process organic acid metabolic process monocarboxylic acid metabolic process small molecule catabolic process

organic acid catabolic process carboxylic acid catabolic process single-organism metabolic process lipid metabolic process fatty acid metabolic process coenzyme metabolic process cofactor metabolic process cellular amino acid catabolic process alpha-amino acid catabolic process acyl-CoA metabolic process thioester metabolic process metabolic process

cellular lipid metabolic process long-chain fatty acid metabolic process organonitrogen compound catabolic process single-organism biosynthetic process

monocarboxylic acid catabolic process aromatic amino acid family catabolic process very long-chain fatty acid metabolic process alpha-amino acid metabolic process organic acid biosynthetic process carboxylic acid biosynthetic process aromatic amino acid family metabolic process small molecule biosynthetic process cellular biogenic amine metabolic process sulfur compound metabolic process

cC

MF $\begin{array}{llllll}0 & 20 & 40 & 60 & 80 & 100\end{array}$

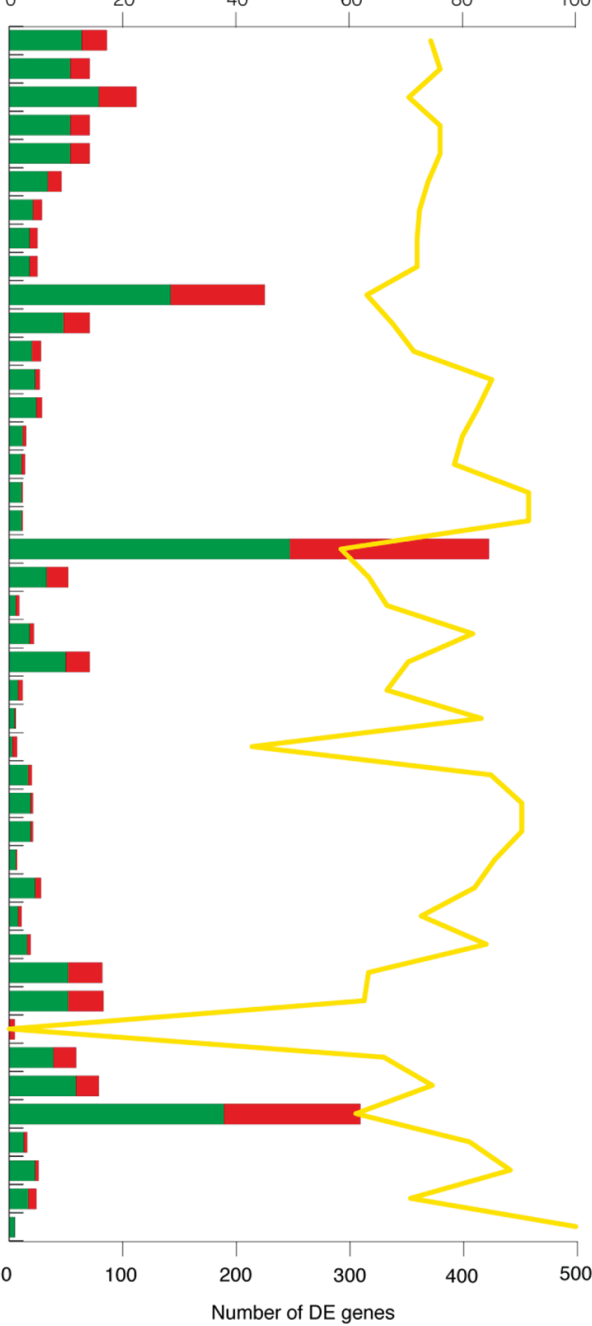

Figure 3. The histogram displaying the gene ontology (GO) terms of differentially expressed genes (DEGs) enrichment significantly (FDR $<0.05$ ) in 4HR0 (A) and 4HR2 (B) group subjected to acute heat stress. In $4 \mathrm{HR} 0$ group, the average of $93.6 \%$ DEGs of enrichment in GO terms were down-regulated. Most of the significant terms were in the BP category. In 4HR0 group, the average of $73.7 \%$ DEGs of enrichment in GO terms were down-regulated. Most of the significant terms were in the BP category. BP:

biological processes, $\mathrm{CC}$ : cellular components and MF: molecular functions 
(a)

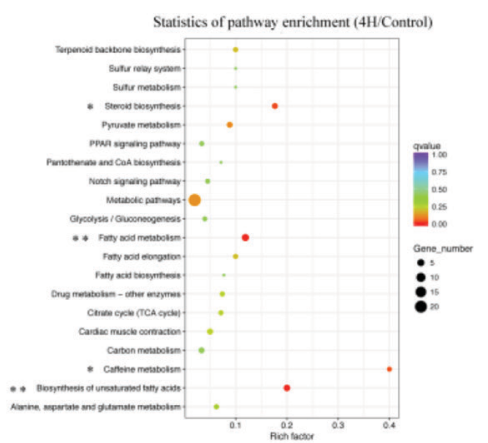

(b)

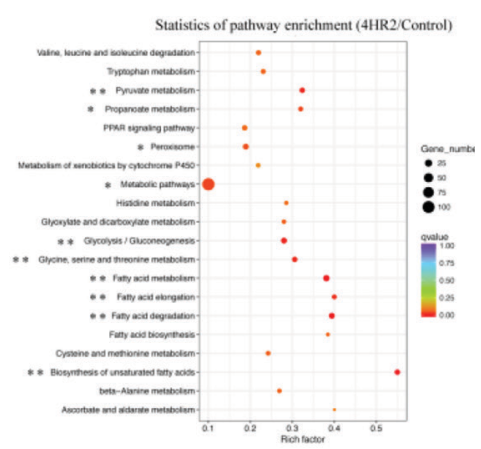

Figure 4. The KEGG pathway enriched analysis significantly $(\mathrm{FDR}<0.05)$ for the differentially expressed genes (DEGs). The asterisk indicates the FDR $<0.05$ and double asterisk indicates the $F D R<0.01$. Rich factor refers to the ratio of the number of differentially enriched genes to the number of annotated genes in the pathway. The rich factor represents the degree of enrichment

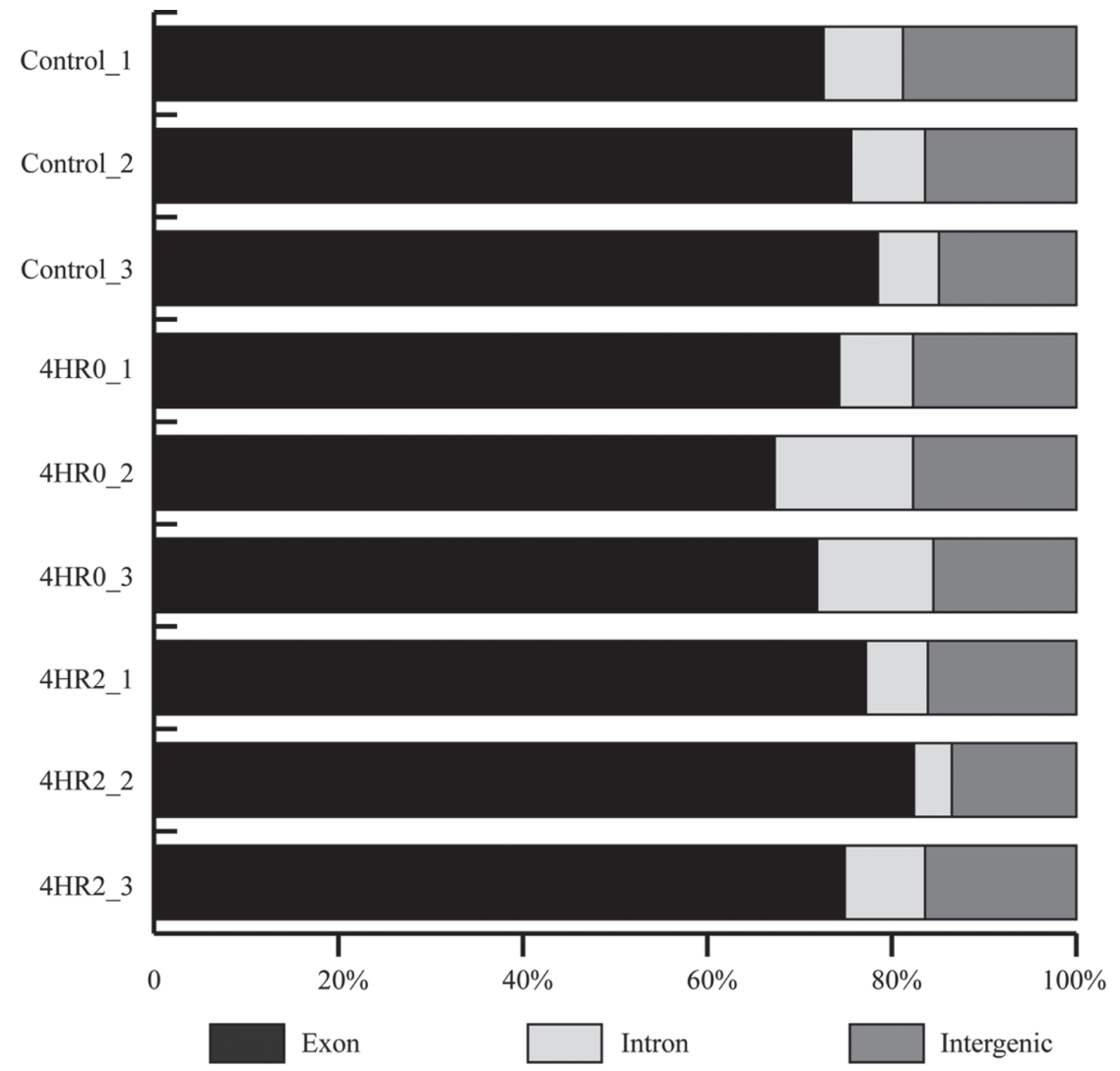

Figure 5. The clean reads were mapped to exons, introns and intergenic genes. 4HR0 group was subjected to acute heat stress at $38^{\circ} \mathrm{C}$ for $4 \mathrm{~h}, 4 \mathrm{HR} 2$ group was subjected to recovery $2 \mathrm{~h}$ at $25^{\circ} \mathrm{C}$ after acute heat stress, and control group was maintained at $25^{\circ} \mathrm{C}$ during the experiment period. There are three biological repetitions for each group 


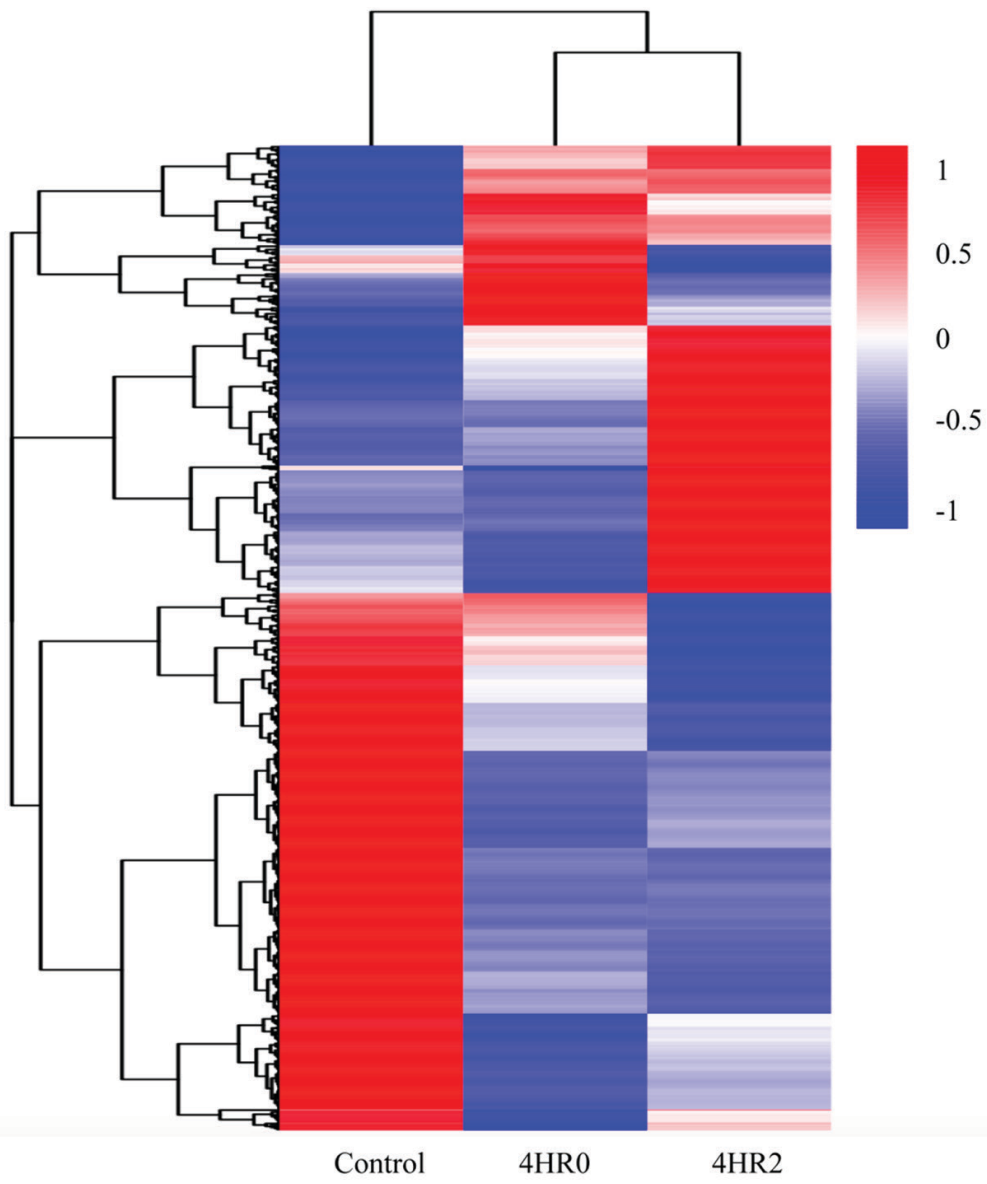

Figure 6. Cluster analysis of differentially expressed genes (DEGs). $\log _{10}(\mathrm{FPKM}+1)$ value normalized transformation was used for the FPKM hierarchical clustering map. Red and blue indicated higher and lower expression genes, respectively

Table 3. Validated DEGs of RNA-seq results by qRT-PCR

\begin{tabular}{l|cc|c|c}
\hline \multirow{2}{*}{ Gene name } & \multicolumn{2}{|c|}{ Log $_{2}$ fold change of RNA-seq } & \multicolumn{2}{c}{$\log _{2}$ fold change of qRT-PCR } \\
\cline { 2 - 5 } & $4 \mathrm{HR} 0$ control & 4HR2/control & $4 \mathrm{HR} 0$ /control & 4HR2/control \\
\hline ACOX1 & 1.41 & $1.33^{* * *}$ & $1.48^{*}$ & $1.42^{*}$ \\
ACACA & -1.65 & $-1.94 * * *$ & $-1.57^{*}$ & $-2.01^{* *}$ \\
ACSL1 & 2.07 & $2.34^{* * *}$ & $1.89^{*}$ & $2.25^{*}$ \\
ACAA1 & 0.59 & $1.87^{* * *}$ & 0.45 & $2.13^{* *}$ \\
FASN & -1.63 & $-2.89^{* * *}$ & -0.98 & $-3.02^{* *}$ \\
HSPA2 & $8.99^{* *}$ & $2.14 * * *$ & $6.38^{* *}$ & $2.46^{* *}$ \\
\hline
\end{tabular}

$\mathrm{n}=6 . * \mathrm{P}<0.05, * * \mathrm{P}<0.01, * * * \mathrm{P}<0.001 .4 \mathrm{HR} 0$ group was subjected to acute heat stress at $38^{\circ} \mathrm{C}$ for $4 \mathrm{~h}, 4 \mathrm{HR} 2$ group was subjected to recovery for $2 \mathrm{~h}$ at $25^{\circ} \mathrm{C}$ after acute heat stress, and control group was maintained at $25^{\circ} \mathrm{C}$ during the experiment period. 


\section{Discussion}

In this study, the rate of change in cloacal temperature after acute heat stress was reported to be slower in yellow-feather chickens compared to that in broilers (May et al., 1987), and the temperature returned to normal after recovery for $2 \mathrm{~h}$, indicating better heat tolerance of the yellow-feather chickens than the commercial broilers.

The physiological response of different breeds to heat stress was reported to be different (Wang et al., 2018). The effect of acute heat stress on the liver transcriptome of broilers was more pronounced than that of chronic heat stress (Lan et al., 2016). The results of previous studies on the liver transcriptome of broilers showed that the DEGs under heat stress were associated with apoptosis, tissue repair, cellular calcium levels (Coble et al., 2014), and immune response (Lan et al., 2016). In this study, the GO and KEGG results show that the response of yellow-feather chickens to heat stress is different from those of the commercial broilers. The response of yellow-feather chickens to heat stress was related apparently more with lipid and energy metabolisms than with physiology.

The DEGs of $A C O X 1, A C A C A, A C S L 1, A C S L 6, A C A A 1, A C A A 2, H A D H B$, and $F A S N$ were associated with fatty acid metabolism. In fatty acid metabolism, $A C A C A$ and $F A S N$, which are associated with the initiation of fatty acid metabolism, were down-regulated in the $4 \mathrm{HR} 2$ group. In chicken, $A C A C A$ encodes a key lipogenesis factor, which has a similar function in mammals (Hillgartner et al., 1996; Takai et al., 1988), and catalyzes the first committed step in the biosynthesis of fatty acids: acetyl-CoA was converted to malonyl-CoA (Abu-Elheiga and Wakil, 2005; Tong, 2005), and the inhibition of ACACA reduces de novo lipogenesis by attenuating the formation of malonyl CoA and long chain fatty acids (Jump et al., 2011), which might play a critical role in the formation of abdominal fat in growing chickens. FASN encodes a key multifunctional enzyme that contributes to the synthesis of fatty acids (Diraison et al., 2002). The mRNA of FASN was mainly expressed in the liver of chicken (Cui et al., 2012), and its expression level was positively correlated with the fat content in the body (Mildner and Clarke, 1991; Nogalska and Swierczynski, 2001). FASN knockout mice die before birth and heterozygous knockout mice die at various stages of development, and indicated that the essential role of FASN is highlighted in the fatty acid biosynthesis (Chirala et al., 2003). ACSL1, ACSL6, $A C O X 1$, and $A C A A 1$ are involved in $\beta$-oxidation of fatty acids. The metabolism of fatty acids, such as the degradation of very long-chain fatty acids, occurs through the $\beta$-oxidation process in the liver (Hashimoto et al., 1999; Veldhoven, 2010). ACSL1 and ACSL6 belong to the acyl-CoA synthetase (ACS) enzyme family (Jia et al., 2007). ACSL1 and ACSL6 prefer long-chain fatty acids (Marszalek et al., 2005; Suzuki et al., 1990) and increased expression of ACSL1 gene is associated with $\beta$-oxidation in liver (Schoonjans et al., 1996). ACSL1 was overexpressed in fastgrowing chickens (Resnyk et al., 2017). ACOX1 acts as the first and rate-limiting enzyme in the $\beta$-oxidation pathway and catalyzes the oxidation of very long-chain fatty acids (VLCFAs) (Fan et al., 1996). This shows that each gene has a different function, which might lead to different fates of fatty acids (Kim et al., 2001; Van Horn et al., 2005). The up-regulation of ACOX1 was reported to stimulate he- 
patic fatty acid oxidation, resulting in excess energy burning in the liver (Misra and Reddy, 2014). These DEGs were also involved in glycolysis/gluconeogenesis and peroxisome pathways. The main roles of liver are: lipid metabolism, glycolysis, and gluconeogenesis. In chickens, liver is more susceptible than other organs to oxidative stress under acute heat stress conditions (Hubbard et al., 2018; Jastrebski et al., 2017; Lin et al., 2006; Tang et al., 2015; Xie et al., 2013).

The results of KEGG analysis showed that the DEGs, ACSS2, ALDH2, ACACA, $D L A T, A L D H 7 A 1, M D H 1$, and $M E 1$ were involved in pyruvate metabolism and $A C S S 2, A C A C A, A B A T, S U C L G 2$, and $A C S S 3$ were involved in propanoate metabolism. The pyruvate and propanoate levels were reported to be associated with energy metabolism (Armentano et al., 1991; Chen et al., 2000; Lam et al., 2005). Pyruvate decreases the intracellular accumulation of triglycerides and under conditions of negative energy balance, liver cells have decreased capacity for converting propionate to glucose (Armentano et al., 1991). Aldehyde dehydrogenases (ALDHs) represent a family of enzymes that catalyze the oxidation of aldehydes to the corresponding carboxylic acids (Vasiliou et al., 2000). ALDH2 is known to catalyze the hydrolysis of esters (Sládek, 2010) and activation of $A L D H 2$ attenuates hepatic injury via clearance of cytotoxic aldehydes (Zhang et al., 2018 a). The ALDH7A1 protein, also known as 'antiquitin,' might have osmoregulatory properties (Vasiliou and Nebert, 2005). And ALDH7A1 catalyzes the terminal step of lysine catabolism, the NAD+dependent oxidation of $\alpha$-aminoadipate semialdehyde to $\alpha$-aminoadipate (Korasick et al., 2017). Short-chain acyl-CoA synthetase (ACSS) is involved in the initial activation step in the metabolism of short-chain fatty acids. ACSS2 and ACSS3 mRNAs are present in various tissues, including liver (Fujino et al., 2000; Yoshimura et al., 2017). The key role of ACSS2 in lipogenesis is to catalyze the synthesis of acetylCoA from acetate (Fujino et al., 2000). Other studies have investigated the underlying mechanism of ACSS2's role in nutrient sensing (Rui et al., 2015). The expression of $A C S S 2$ gene was reported to be involved in fatty acid synthesis, triacylglycerol synthesis, and fatty acid oxidation (Xu et al., 2018). Moreover, ACSS2 has a higher affinity for propionate (Ingram, 2014). The role of ACSS2 was in promoting cell growth and survival in nutrient-stressed conditions (Dodhia et al., 2017). ACSS3 is present in the mitochondrial matrix of liver and has high affinity for propionic acid, and its expression is up-regulated under ketogenic conditions (Yoshimura et al., 2017). ACSS3 is related to the propanoate metabolism, and catalyzes the conversion of propanoyl-CoA to propanoate (Buitenhuis et al., 2014). DLAT catalyzes the conversion of pyruvate to acetyl-CoA, presumably to facilitate oxidative phosphorylation, ATP generation, and catabolic reactions (Goh et al., 2015). The important roles of the essential malate dehydrogenase, i.e., mitochondrial malate dehydrogenase $(\mathrm{MDH} 2)$, is to produce energy through aerobic respiration in the Krebs cycle. In addition, $\mathrm{MDH} 2$ is associated with de novo lipid synthesis (Bourneuf et al., 2006; Schmid et al., 2010). Knockdown of DLAT reduced cell proliferation by approximately $20-45 \%$ that presumably resulted in decrease in energy production (Goh et al., 2015). ME1 (malic enzyme) is a NADP-dependent lipase, which is associated with the conversion of L-malate to pyruvate. ME1 is linked between the glycolytic pathway and the Krebs cycle through acetyl transfers (Macdonald, 1995). Moreover, 
MDH2 and ME1 are involved in the synthesis and secretion of lipids (Bourneuf et al., 2006; Zhou et al., 2012). The expression levels of ME1 in chickens with fatty liver were higher than those in chickens without fatty liver (Zhang et al., 2018 b).

In summary, the results provide insights into the transcriptomic regulation of acute heat stress in the liver of yellow-feather chickens. A total of 834 DEGs were detected under heat stress. These were found to be involved in the lipid and energy metabolism, including fatty acid metabolism, pyruvate metabolism, and propanoate metabolism. Our findings provide the context for RNA-seq studies in the liver of yellow-feather chickens and suggest that the liver of yellow-feather broilers has the lipid and energy metabolism physiological mechanisms activated in response to heat stress.

\section{Acknowledgments}

The work was supported by the Guangdong Ocean University Research System (101402/R17030, CXXL2018148), and Open Fund of Guangdong Provincial Key Laboratory of Agro-animal Genomics and Molecular Breeding, South China Agricultural University (201702).

\section{Conflict of interest}

We certify that there is no conflict of interest with any financial organization regarding the material discussed in the manuscript.

\section{References}

A b u - E 1 h e ig a L., Wakil S.J. (2005). Mutant mice lacking acetyl-CoA carboxylase 1 are embryonically lethal. Proc. Natl. Acad. Sci. USA, 102: 12011-12016.

Anders S., Huber W. (2010). Differential expression analysis for sequence count data. Genome Biol., 11: 1-12.

A n d e r s S., P y 1 P.T., H u b e r W. (2015). HTSeq-a Python framework to work with high-throughput sequencing data. Bioinformatics, 31: 166-169.

A r m e nt a no L.E., Grum mer R.R., B ertic s S.J., S k a ar T.C., D on kin S.S. (1991). Effects of energy balance on hepatic capacity for oleate and propionate metabolism and triglyceride secretion. J. Dairy Sci., 74: 132-139.

B ourneuf E., Hérault F., Ch i c a ult C., Carré W., A s saf S., Monnier A., Mottier S., Lagarrigue S., Dou aire M., Mosser J. (2006). Microarray analysis of differential gene expression in the liver of lean and fat chickens. Gene, 372: 162-170.

B u it e nhu is B., Jans s L.L., P ou 1 sen N.A., Larsen L.B., L ars en M.K., Søren sen P. (2014). Genome-wide association and biological pathway analysis for milk-fat composition in Danish Holstein and Danish Jersey cattle. BMC Genomics, 15: 1112.

$\mathrm{C}$ a h a n e r A., L e e n str a F. (1992). Effects of high temperature on growth and efficiency of male and female broilers from lines selected for high weight gain, favorable feed conversion, and high or low fat content. Poultry Sci., 71: 1237-1250.

Chen C.J., Cheng F.C., Li a o S.L., Chen W.Y., L in N.N., K u o J.S. (2000). Effects of naloxone on lactate, pyruvate metabolism and antioxidant enzyme activity in rat cerebral ischemia/reperfusion. Neurosci. Lett., 287: 113-116.

Cheng C., Tu W., Chen C., Chan H., Chen C., Chen H., Tang P., Lee Y.P., Chen S., $\mathrm{Hu}$ ang S. (2018). Functional genomics study of acute heat stress response in the small yellow follicles of layer-type chickens. Sci. Rep., 8: 1320. 
Chirala S.S., Hua C., Matzuk M., A bu - Elhe iga L., Ma o J., Mahon K., Fin e gold M., Wakil S.J. (2003). Fatty acid synthesis is essential in embryonic development: fatty acid synthase null mutants and most of the heterozygotes die in utero. Proc. Natl. Acad. Sci. USA, 100: 6358-6363.

Coble D.J., Fleming D., Persia M.E., A shwell C.M., Roth s child M.F., S chmidt C.J., L a m o n t S.J. (2014). RNA-seq analysis of broiler liver transcriptome reveals novel responses to high ambient temperature. BMC Genomics, 15: 1084.

Cu i H.X., Z he ng M.Q., L i u R.R., Z h a o G.P., Ch e n J.L., We n J. (2012). Liver dominant expression of fatty acid synthase (FAS) gene in two chicken breeds during intramuscular-fat development. Mol. Biol. Rep., 39: 3479.

D e e b N., C a ha ner A. (2002). Genotype-by-environment interaction with broiler genotypes differing in growth rate. 3. Growth rate and water consumption of broiler progeny from weight-selected versus nonselected parents under normal and high ambient temperatures. Poultry Sci., 81: 293-301.

Diraison F., Dusserre E., Vidal H., Sothier M., B eylot M. (2002). Increased hepatic lipogenesis but decreased expression of lipogenic gene in adipose tissue in human obesity. Am. J. Physiol.-Endocrinol. Metab., 282: 46-51.

Dodhia S., Celis K., Aylward A., Cai Y., Fontana M.E., Trespalacios A., Hoffman D.C., A 1 fon s o H.O., E is i g S.B., S u G.H. (2017). ACSS2 gene variant associated with cleft lip and palate in two independent Hispanic populations. Laryngoscope, 127: 336-339.

F a n C.Y., P a n J., Chu R., L e e D., K l u c k man K.D., Us u d a N., S ingh I., Yeld andi A.V., R a o M.S., Ma e d a N. (1996). Hepatocellular and hepatic peroxisomal alterations in mice with a disrupted peroxisomal fatty acyl-coenzyme A oxidase gene. J. Biol. Chem., 271: 24698-24710.

F uj in o T., S a t o H., Cho Y., Y a m a m o to T.T. (2000). Molecular characterization of short-chain acyl-CoA synthetases. In: Lipoprotein Metabolism and Atherogenesis, Kita T., Yokode M. (eds). Springer, Tokyo, Japan.

Goh W.Q., Ow G.S., Kuznet s o v V.A., Chong S., Li m Y.P. (2015). DLAT subunit of the pyruvate dehydrogenase complex is upregulated in gastric cancer-implications in cancer therapy. Am. J. Transl. Res., 7: 1140-1151.

Ha sh i moto T., F uj i t a T., Us u d a N., Cook W., Q i C., P eters J.M., Gonzal ez F.J., Ye 1 d a n d i A.V., R a o M.S., Reddy J.K. (1999). Peroxisomal and mitochondrial fatty acid betaoxidation in mice nullizygous for both peroxisome proliferator-activated receptor alpha and peroxisomal fatty acyl-CoA oxidase. Genotype correlation with fatty liver phenotype. J. Biol. Chem., 274: 19228-19236.

Hillgartner F.B., Charron T., Che snut K.A. (1996). Alterations in nutritional status regulate acetyl-CoA carboxylase expression in avian liver by a transcriptional mechanism. Biochem. J., 319 : 263-268.

$\mathrm{H}$ u a $\mathrm{n}$ g S. (2017). Upregulation of TLR4 mRNA expression levels in broiler chiekens under acute heat stress. Braz. J. Poult. Sci., 19: 87-94.

Hubbard A., Zhang X., J a s trebski S., Singh A., S chmidt C. (2018). Understanding the liver under heat stress with statistical learning: a multiomics computational approach. BioRxiv, 340125.

In g r a m C. (2014). AMP-forming acetyl-CoA synthetases in Archaea show unexpected diversity in substrate utilization. Archaea, 2: 95-107.

J a s trebski S.F., La m on t S.J., S c h m id t C.J. (2017). Chicken hepatic response to chronic heat stress using integrated transcriptome and metabolome analysis. PloS One, 12: e0181900.

J i a Z., M ou l s o n C.L., P e i Z., M in e r J.H., Watk in s P.A. (2007). FATP4 is the principal very long-chain fatty acyl-CoA synthetase in skin fibroblasts. J. Biol. Chem., 21: A607-A608.

J u m p D.B., T o r re s - G o n z a le z M., O l s o n L.K. (2011). Soraphen A, an inhibitor of acetyl CoA carboxylase activity, interferes with fatty acid elongation. Biochem. Pharmacol., 81: 649-660.

K a n e h is a M., G o t o S. (1999). KEGG: Kyoto Encyclopedia of Genes and Genomes. Nucleic Acids Res., 28: 27-30.

K i m D., L a n g m e a d B., S a 1 z b e r g S.L. (2015). HISAT: a fast spliced aligner with low memory requirements. Nat. Methods., 12: 357-360.

K i m J.H., L e w in T.M., C o 1 e m a n R.A. (2001). Expression and characterization of recombinant rat Acyl-CoA synthetases 1, 4, and 5. Selective inhibition by triacsin $\mathrm{C}$ and thiazolidinediones. J. Biol. Chem., 276: 24667. 
Korasick D.A., Wyatt J.W., Luo M., Laciak A.R., Ruddraraju K., Gates K.S., Hen z l M.T., T a n n e r J.J. (2017). Importance of the C-terminus of aldehyde dehydrogenase 7al for oligomerization and catalytic activity. Bioinformatics, 56: 5910-5919.

Lam T.K., Gutierrez-Juarez R., P oc ai A., Ross etti L. (2005). Regulation of blood glucose by hypothalamic pyruvate metabolism. Science, 309: 943-947.

Lan X., Hsieh J.C.F., S chmidt C.J., Zhu Q., Lamont S.J. (2016). Liver transcriptome response to hyperthermic stress in three distinct chicken lines. BMC Genomics, 17: 955.

L in H., D e cuypere E., Buys e J. (2006). Acute heat stress induces oxidative stress in broiler chickens. Comp. Biochem. Physiol. A: Mol. Integr. Physiol., 144: 11-17.

Livak K.J., S chmittgen T.D. (2001). Analysis of relative gene expression data using real-time quantitative PCR and the 2(-Delta Delta C(T)) Method. Methods, 25: 402-408.

L u Q., Wen J., Z h a n g H. (2007). Effect of chronic heat exposure on fat deposition and meat quality in two genetic types of chicken. Poultry Sci., 86: 1059-1064.

Ma c d o nald M.J. (1995). Feasibility of a mitochondrial pyruvate malate shuttle in pancreatic islets. Further implication of cytosolic NADPH in insulin secretion. J. Biol. Chem., 270: 20051-20058.

Ma o X., Cai T., Oly archuk J.G., We i L. (2005). Automated genome annotation and pathway identification using the KEGG Orthology $(\mathrm{KO})$ as a controlled vocabulary. Bioinformatics, 21 : 3787-3793.

Mars za le k J.R., K i t i d is C., D i ru s s o C.C., L o d i s h H.F. (2005). Long-chain acyl-CoA synthetase 6 preferentially promotes DHA metabolism. J. Biol. Chem., 280: 10817-10826.

May J.D., D e a to n J.W., B ran to n S.L. (1987). Body temperature of acclimated broilers during exposure to high temperature. Poultry Sci., 66: 378-380.

Mcka y J.C., B arton N.F., K o e rhu is A.N.M., M c a d a m J. (2000). Editors. The challenge of genetic change in the broiler chicken. Edinburgh, UK, British Society of Animal Science Press, $86 \mathrm{pp}$.

M i l d n e r A.M., C 1 a r k e S.D. (1991). Porcine fatty acid synthase: cloning of a complementary DNA, tissue distribution of its mRNA and suppression of expression by somatotropin and dietary protein. J. Nutr., 121: 900.

Mis ra P., Reddy J.K. (2014). Peroxisome proliferator-activated receptor- $\alpha$ activation and excess energy burning in hepatocarcinogenesis. Biochimie. 98: 63-74.

N o ga l s k a A., S w i e r c z y n s ki J. (2001). The age-related differences in obese and fatty acid synthase gene expression in white adipose tissue of rat. Biochim. Biophys. Acta Mol. Cell Biol. Lipids, 1533: 73-80.

Resnyk C.W., Carré W., Wang X., Porter T.E., Simon J., Bihan-Duval E.L., Duc los M.J., A g g re y S.E., C o g burn L.A. (2017). Transcriptional analysis of abdominal fat in chickens divergently selected on bodyweight at two ages reveals novel mechanisms controlling adiposity: validating visceral adipose tissue as a dynamic endocrine and metabolic organ. BMC Genomics, 18: 626.

Rimold i S., L a s a g n a E., S a rt i F.M., Marelli S.P., Cozzi M.C., B ernard in i G., Te ro v a G. (2015). Expression profile of six stress-related genes and productive performances of fast and slow growing broiler strains reared under heat stress conditions. Meta Gene, 6: 17-25.

R u i C., M in X., N a gat i J.S., Hogg R.T., A lok D., G e rard R.D., G a r c i a J.A. (2015). The acetate/ACSS2 switch regulates HIF-2 stress signaling in the tumor cell microenvironment. Plos One, 10: e0116515.

S c h m i d G.M., C onvers e t V., Wal t e r N., S e nn it t M.V., L e ung K.Y., B y e r s H., Ward M., Hochstrasser D.F., Caw thorne M.A., S anchez J.C. (2010). Effect of high-fat diet on the expression of proteins in muscle, adipose tissues, and liver of C57BL/6 mice. Proteomics, 4 : $2270-2282$.

Schoonjans K., Staels B., A uw erx J. (1996). Role of the peroxisome proliferator-activated receptor (PPAR) in mediating the effects of fibrates and fatty acids on gene expression. J. Lipid Res., 37: 907-925.

Schurch N.J., S chofield P., Gierliński M., Cole C., Sherstnev A., S ingh V., Wrobel N., Gharbi K., Simps on G.G., Owen-Hughes T., Blaxter M., B arton G.J. (2016). How many biological replicates are needed in an RNA-seq experiment and which differential expression tool should you use? RNA, 22: 839-851.

S lá d e k N.E. (2010). Human aldehyde dehydrogenases: Potential pathological, pharmacological, and toxicological impact. J. Biochem. Mol. Toxicol., 17: 7-23. 
Sohail M.U., Hume M.E., Byrd J.A., N isbet D.J., Ijaz A., Sohail A., Shabbir M.Z., R ehman H. (2012). Effect of supplementation of prebiotic mannan-oligosaccharides and probiotic mixture on growth performance of broilers subjected to chronic heat stress. Poultry Sci., 91: $2235-2240$.

Suzuki H., Kawarabayas i Y., Kond o J., Abe T., Nishikawa K., Ki mura S., Hashim o to T., Y a m a m o to T. (1990). Structure and regulation of rat long-chain acyl-CoA synthetase. J. Biol. Chem., 265: 8681-8685.

Tak a i T., Yo k o y a m a C., Wa d a K., T a n a be T. (1988). Primary structure of chicken liver acetylCoA carboxylase deduced from cDNA sequence. J. Biol. Chem., 263: 2651-2657.

Tang X., Meng Q., G a o J., Zhang S., Zhang H., Zhang M. (2015). Label-free quantitative analysis of changes in broiler liver proteins under heat stress using SWATH-MS technology. Sci. Rep., 5: 15119.

To n g L. (2005). Acetyl-coenzyme A carboxylase: crucial metabolic enzyme and attractive target for drug discovery. Cell. Mol. Life Sci., 62: 1784-1803.

Trapnell C., Williams B.A., Pertea G., Mortazavi A., Kwan G., Van Baren M.J., S a lzberg S.L., Wold B.J., P a chter L. (2010). Transcript assembly and quantification by RNA-Seq reveals unannotated transcripts and isoform switching during cell differentiation. Nat. Biotechnol., 28: 511-515.

Van Horn C.G., C a vig li a J.M., Li L.O., Wang S., Granger D.A., Cole man R.A. (2005). Characterization of recombinant long-chain rat acyl-CoA synthetase isoforms 3 and 6: identification of a novel variant of isoform 6. Biochemistry, 44: 1635-1642.

Vasili ou V., Nebert D.W. (2005). Analysis and update of the human aldehyde dehydrogenase (ALDH) gene family. Hum. Genom., 2: 138-143.

Vasili o u V., P a p p a A., P e ters en D.R. (2000). Role of aldehyde dehydrogenases in endogenous and xenobiotic metabolism. Chem. Biol. Interact., 129: 1-19.

Veld hove n P.P.V. (2010). Biochemistry and genetics of inherited disorders of peroxisomal fatty acid metabolism. J. Lipid Res., 51: 2863-2895.

Wang Y., Saelao P., Chanthavixay K., Gallardo R., Bunn D., Lamont S.J., Dekk e r s J.M., K elly T., Z h o u H. (2018). Physiological responses to heat stress in two genetically distinct chicken inbred lines. Poultry Sci., 97: 770-780.

W ind horst H.W. (2007). Changes in poultry production and trade worldwide. World's Poultry Sci. J., 62: 585-602.

X i e J., Li T., L in L., Z ha ng L., L in X., L i u H.C., O d l e J., L u o X. (2013). Differential expression of heat shock transcription factors and heat shock proteins after acute and chronic heat stress in laying chickens (Gallus gallus). PloS One, 9: e102204.

Xu H., Luo J., Ma G., Zhang X., Yao D., Li M., Loor J.J. (2018). Acyl-CoA synthetase short-chain family member 2 (ACSS2) is regulated by SREBP-1 and plays a role in fatty acid synthesis in caprine mammary epithelial cells. J. Cell. Physiol., 233: 1005-1016.

Yoshimura Y., Araki A., Maruta H., Takahashi Y., Yamashita H. (2017). Molecular cloning of rat acss 3 and characterization of mammalian propionyl-CoA synthetase in the liver mitochondrial matrix. J. Biochem., 161: 279-289.

Young M.D., Wakefield M.J., S m y th G.K., O shla ck A. (2010). Gene ontology analysis for RNA-seq: accounting for selection bias. Genome Biol., 11: 1-12.

Zhang Q., Zhang B., Lu o Y. (2019). Cardiac transcriptome study of the effect of heat stress in yellow-feather broilers. Ital J. Anim. Sci., 18: 971-975.

Zhang T., Zhao Q., Ye F., Huang C.Y., Chen W.M., Huang W.Q. (2018 a). Alda-1, an ALDH2 activator, protects against hepatic ischemia/reperfusion injury in rats via inhibition of oxidative stress. Free Radic. Res., 52: 629-638.

Zhang Y., Liu Z., Li u R., Wang J., Zheng M., Li Q., Cui H., Zh a o G., Wen J. (2018 b). Alteration of hepatic gene expression along with the inherited phenotype of acquired fatty liver in chicken. Genes, 9: 199.

Z h o u S.L., Li M.Z., L i Q.H., Gu an J.Q., Li X.W. (2012). Differential expression analysis of porcine MDH1, MDH2 and ME1 genes in adipose tissues. Genet. Mol. Res., 11: 1254-1259.

Received: 9 IV 2019

Accepted: 11 IX 2019 